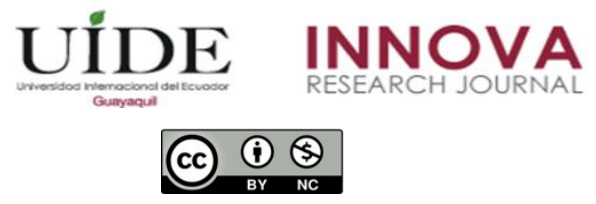

INNOVA Research Journal, ISSN 2477-9024

(Septiembre-Diciembre 2020). Vol. 5, No.3 pp. 196-208

DOI: https://doi.org/10.33890/innova.v5.n3.2020.1410

URL: http://revistas.uide.edu.ec/index.php/innova/index

Correo: innova@uide.edu.ec

\title{
Evaluación del Impacto de Diversos Factores Macroeconómicos en el Crecimiento Económico del Ecuador, 1965-2018
}

\section{Evaluation of the Impact of Various Macroeconomic Factors on the Economic Growth of Ecuador, 1965-2018}

Jorge Antonio Ruso León

https://orcid.org/0000-0001-9505-8237

Edmundo Ricardo Contreras Chacón

https://orcid.org/0000-0001-5217-5521

Digna Priscila Villamar Ortiz

https://orcid.org/0000-0002-4601-8674

Universidad Agraria del Ecuador, Ecuador

Autor para correspondencia: jruso@uagraria.edu.ec; econtreras@uagraria.edu.ec; pvillamar@uagraria.edu.ec

Fecha de recepción: 24 de abril del 2020 - Fecha de aceptación: 10 de agosto del 2020

\section{Resumen}

La medición del crecimiento económico, a través de su principal indicador, el Producto Interno Bruto, es fundamental para los responsables de la política económica de un país. Existen tres formas diferentes de calcular el Producto Interno Bruto, una de ellas es el método de gasto que incluye en su cálculo cinco importantes variables macroeconómicas. Esta investigación tiene como objetivo cuantificar la incidencia de cada una de esas variables en el crecimiento económico del Ecuador. Para ello, utilizando la información correspondiente a esas variables para el período 1965-2018, se estimó, mediante una regresión lineal múltiple por mínimos cuadrados ordinarios, la ecuación para el cálculo del Producto Interno Bruto por el método de gasto, una vez que ésta fue validada estadísticamente se analizaron los coeficientes estandarizados de cada variable macroeconómica involucrada en la misma para evaluar cuán determinante es para el Producto Interno Bruto del Ecuador. Se demostró que el consumo es la variable que mayor peso tiene para generar demanda agregada, seguida de las exportaciones, contrariamente la inversión se revela como la variable macroeconómica que menos incide en el crecimiento económico ecuatoriano. Como conclusiones fundamentales se determinó que el consumo es el principal determinante del crecimiento económico del Ecuador por lo que políticas que lo reduzcan provocan directamente una afectación sobre el crecimiento económico, asimismo, se halló que la inversión es el componente que menos impacta lo cual implica la necesidad de que se diseñen políticas que la incentiven.

Palabras Claves: PIB; regresión lineal múltiple; MCO; método de gasto; crecimiento económico 


\begin{abstract}
The measurement of economic growth, through its main indicator, the Gross Domestic Product, is essential for those responsible for the economic policy of a country. There are three different ways of calculating the Gross Domestic Product, one of them is the spending method that includes five important macroeconomic variables in its calculation. This research aims to quantify the incidence of each of these variables in the economic growth of Ecuador. For this, using the information corresponding to these variables for the period 1965-2018, the equation for the calculation of the Gross Domestic Product by the expenditure method was estimated, by means of a multiple linear regression by ordinary least squares, once it was statistically validated, the standardized coefficients of each macroeconomic variable involved in it were analyzed to assess how decisive it is for the Gross Domestic Product of Ecuador. It was shown that consumption is the variable that has the greatest weight to generate aggregate demand, followed by exports, conversely, investment is revealed as the macroeconomic variable that has the least impact on Ecuadorian economic growth. As fundamental conclusions, it was determined that consumption is the main determinant of economic growth in Ecuador, so that policies that reduce it directly affect economic growth, and it was also found that investment is the component that least impacts, which implies need to design policies that encourage it.
\end{abstract}

Key Words: GDP; multiple linear regression; OLS; spending method; economic growth

\title{
Introducción
}

La medición de la actividad económica es una actividad fundamental que deben llevar a cabo los responsables de la política económica en todos los países para vigilar las tendencias de los diferentes indicadores y adoptar las medidas de corrección de manera oportuna.

Entre los indicadores fundamentales que deben ser objeto de monitoreo constante se encuentra el Producto Interno Bruto (PIB), pues este, como indicativo de la renta total del país y del gasto total en la producción de bienes y servicios, según (Mankiw N. G., 2014), suele considerarse como el mejor indicador de los resultados de la economía, asimismo, puede afirmarse, citando a (Abel \& Bernanke, 2004), que el PIB es el indicador más amplio de la actividad económica agregada, así como el más conocido y utilizado.

Por otra parte, y a tenor de (Dornbusch, Fischer, \& Startz, 2009), los datos del PIB no solo sirven como medida de cuanto se produce, sino también como medida del bienestar de los habitantes de un país. También debe decirse que el PIB, tampoco es una medida perfecta, completa y exacta del cambio en el bienestar económico de una nación, pues existen factores que este indicador no mide o que no mide con exactitud, a partir de lo planteado por (Parkin, Esquivel, \& Muñoz, 2007), lo cual, a pesar de todo, no limita su utilidad.

Teniendo en cuenta, entonces, que (Kiziryan, 2019), define al PIB como el total de bienes y servicios demandados por un país, a un determinado nivel de precios, en un determinado periodo de tiempo y que, según (Gastón Lorente, 2019), este indicador resume en una única cifra el valor monetario de la actividad económica en un determinado periodo de tiempo convirtiéndose en un reflejo de la evolución económica de un país y del ritmo al que se incrementa su producción de bienes y servicios y, por tanto, su renta, es útil conocer cómo o en 
qué medida impactan en la evolución del PIB los elementos o factores macroeconómicos que lo determinan.

Por ello, el objetivo general que se plantea esta investigación es:

- Cuantificar el impacto de los factores macroeconómicos determinantes del crecimiento económico en el Ecuador, tomando como referencia el método del gasto para el cálculo del PIB.

Los objetivos específicos que guiarán la investigación hacia el objetivo general son:

- Describir el comportamiento general de las series estudiadas, así como, evaluar sus propiedades de estacionariedad y cointegración.

- Estimar el modelo econométrico, a partir del método de gasto, que describe el PIB del Ecuador y los factores que lo explican para el período de análisis.

- Validar los supuestos del modelo econométrico hallado.

\section{Marco teórico}

Revisando a (Mankiw, 2014), este afirma que el producto interior bruto o PIB suele considerarse el mejor indicador de los resultados de la economía. También plantea que, el objetivo del PIB es resumir todos los datos económicos en una única cifra que representa el valor monetario de la actividad económica en un determinado periodo de tiempo.

Citando a (Samuelson \& Nordhaus, 2010), queda claro que el PIB se utiliza para muchos fines, pero el más importante es medir el desempeño global de una economía.

Para (Case \& Fair, 2008), el PIB se calcula de dos maneras. Una consiste en sumar el total gastado en todos los bienes finales durante cierto periodo. Éste es el método del gasto para calcular el PIB. La otra es sumar el ingreso (salarios, rentas, intereses y utilidades) que recibieron todos los factores de producción al manufacturar los bienes finales. Es el método del ingreso para calcular el PIB. Los dos métodos llevan a la misma cifra del PIB pues: todo pago (gasto) de un comprador es, al mismo tiempo, una entrada (ingreso) del vendedor. Podemos medir los ingresos recibidos o los gastos incurridos, y terminaremos con la misma producción total.

Por su parte, (Abel \& Bernanke, 2004) expresan que, la cantidad de actividad económica que se realiza en un período de tiempo puede medirse por medio de:

1. La cantidad de producción, excluida la que se utiliza en las fases intermedias de producción (el método del producto).

2. La renta percibida por los productores (el método de la renta).

3. La cantidad de gasto de los compradores últimos de la producción (el método del gasto).

Cada uno de estos métodos ofrece una perspectiva diferente de la economía. Sin embargo, el principio fundamental en el que se basan todos es que, salvando algunos problemas 
como los datos incompletos o mal declarados, los tres métodos generan medidas idénticas de la cantidad de actividad económica (Abel \& Bernanke, 2004).

Tomando como referencia la explicación que aportan (González González, y otros, 2009) sobre el flujo circular de la actividad económica, éste representa las condiciones generales para que una economía encuentre el equilibrio. Estas condiciones son que la producción sea igual al gasto y a la renta.

El modelo del flujo circular del gasto y el ingreso, según (Parkin, Esquivel, \& Muñoz, 2007), demuestra que el gasto agregado es igual al ingreso agregado lo cual es el fundamento que explica por qué los tres métodos antes enunciados deben generar el mismo resultado para el PIB.

Por ello, en la presente investigación nos centraremos en analizar uno de los tres métodos, en este caso, el método de gasto, teniendo en cuenta los factores macroeconómicos que integran su cálculo y cómo y en qué medida éstos son determinantes para el PIB y por ende para el crecimiento económico.

Ahora bien, cuáles son los indicadores macroeconómicos que integran el cálculo del PIB por el método de gasto, si revisamos a (Gastón Lorente, 2019), éste expresa que el PIB hay tres formas de calcularlo, una de ellas es el método del gasto, que se formula de la siguiente manera:

$$
P I B=C+I+G+X-M
$$

Donde, "C" son los bienes y servicios demandados para consumo, "I" para inversión, "G" por el gobierno y " $\mathrm{X}$ " los bienes y servicios exportados e "M" importados.

Citando a (Jahan, Saber Mahmud, \& Papageorgiou, 2014), se puede plantear que el producto de bienes y servicios de una economía es la suma de cuatro componentes: consumo, inversión, compras del gobierno y las exportaciones netas, entonces cualquier variación del PIB tiene que provenir de uno de esos cuatro componentes. Es por ello que se estudiará en la presente investigación y, basados en los datos empíricos, cómo estos elementos inciden en el crecimiento económico del Ecuador.

\section{Metodología}

El enfoque de la presente investigación es cuantitativo y desde el punto de vista de su alcance lo definimos como correlacional en tanto que, se pretende establecer la relación existente entre el PIB del Ecuador y las diferentes variables que lo determinan.

Para proceder con el desarrollo del estudio se comenzaría compilando la información del período 1965-2018 de las siguientes variables para el Ecuador:

Variable dependiente o explicada.

- Producto Interno Bruto. (PIB) 


\section{Variables independientes o explicativas.}

- Consumo. (C)

- Inversión. (I)

- Gasto fiscal. (G)

- Exportaciones. (X)

- Importaciones. (M)

Esta información es pública y está disponible en (Banco Central del Ecuador, 2020).

Para cada variable se obtuvieron 54 observaciones con frecuencia anual, correspondientes al período de 1965 al 2018, los datos se tabularon y graficaron, ver la figura 1, lo que permitiría analizar la tendencia, el ajuste y el comportamiento en general de todas las variables involucradas en el estudio.

Luego se analizaría la dimensión de las variables, todas ellas expresadas en miles de dólares y teniendo en cuenta también que se quería obtener la relación natural entre las variables se decidió descartar cualquier transformación del tipo Box-Cox, (Lind, Marchal, \& Wathen, 2012), por lo que se estimaría un modelo de regresión lineal múltiple en su forma funcional lineal-lineal, el que se describe a continuación, según (Anderson, Sweeney, \& Williams, 2008):

$$
\hat{y}_{t}=\widehat{\beta_{0}}+\widehat{\beta_{1}} x_{1 t}+\widehat{\beta_{2}} x_{2 t}+\cdots+\widehat{\beta_{p}} x_{p t}
$$

donde:

$\hat{y}_{t}$ : es el valor estimado de la variable dependiente en el momento $t$.

$x_{p t}$ : es el valor de la variable independiente $p$ en el momento $t$.

$\widehat{\beta_{p}}$ : es el valor estimado del coeficiente de la variable independiente $p$.

Debido a que todas las variables a utilizar en el modelo de regresión a estimar son series de tiempo de indicadores económicos existe el riesgo de obtener una regresión espuria, (Gujarati \& Porter, 2010), para evitar este problema, independientemente de lo que la teoría postula sobre la real relación de estas variables, se debería determinar la estacionariedad o no estacionariedad de las series de tiempo estudiadas y su orden de integración, para ello se realizaría la prueba de Dickey -Fuller Aumentada $\left(A D F^{1}\right)$ a cada una de las variables en niveles y en diferencias, si dichas variables resultaran ser series no estacionarias y con igual número de raíces unitarias se pasaría a ejecutar el contraste de Cointegración de Engle-Granger, (Court \& Williams, 2011), entre las mismas con el fin de comprobar si pudiera existir una relación espuria entre ellas.

En cuanto a probar estacionariedad con la prueba $A D F$, teniendo en cuenta lo planteado por (Mahadeva \& Robinson, 2009) respecto a que esta prueba en ocasiones puede llevar a cometer el error de tipo II al aceptar una hipótesis nula siendo esta falsa, utilizaríamos, en caso de duda, la prueba $K P S S^{2}$ para confirmar un resultado dudoso de la prueba $A D F$.

\footnotetext{
1 Augmented Dickey-Fuller

${ }^{2}$ Kwiatkowski-Phillips-Schmidt-Shin
} 
La ecuación de regresión se estimaría realizando una regresión lineal múltiple por $\mathrm{MCO}^{3}$ a las series de datos para el período comprendido entre 1965 y 2018.

\section{Figura 1}

Evolución de las variables estudiadas en el período desde 1965 al 2018.

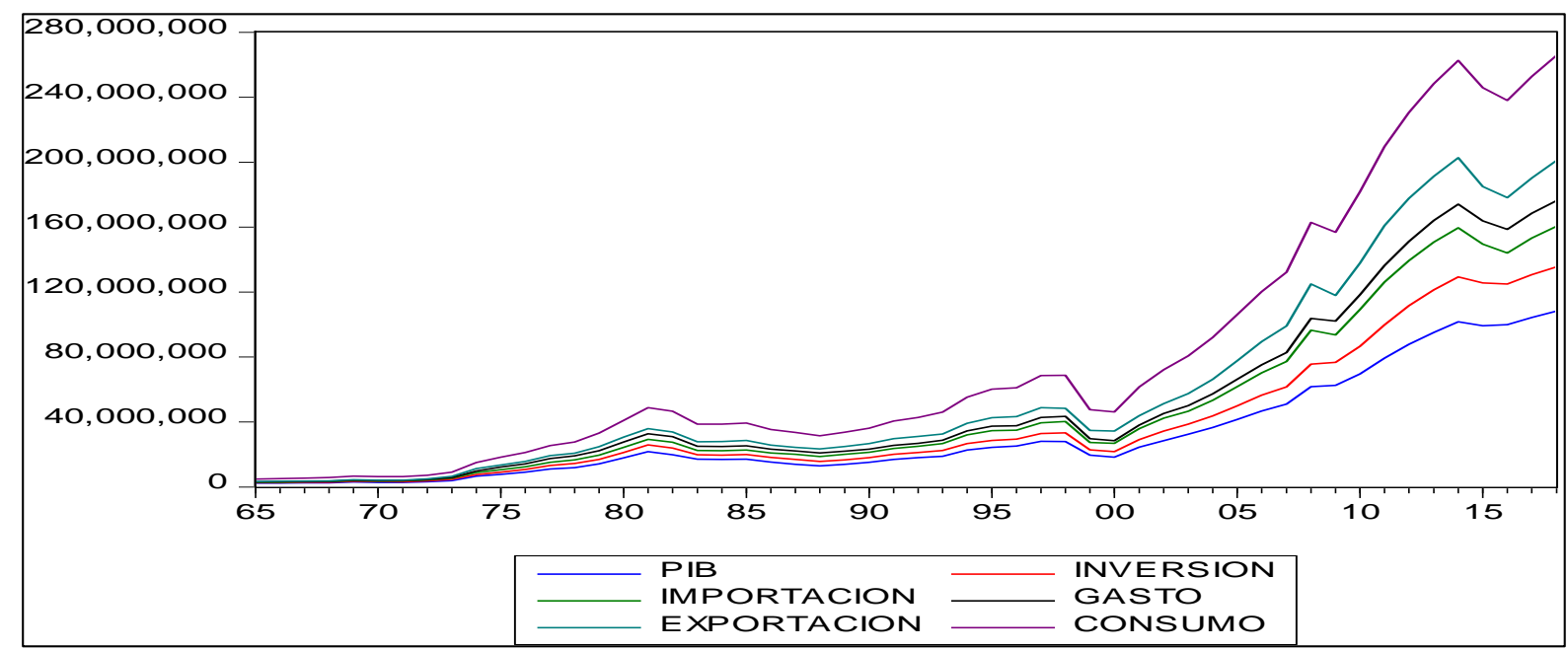

Fuente: Banco Central del Ecuador (2020).

Luego de estimada la ecuación de regresión se procedería a verificar la significancia global e individual de los estimadores obtenidos mediante las respectivas pruebas $F$ y $t$.

A continuación, se procedería a ejecutar el proceso de validación de los supuestos de la regresión lineal para la ecuación obtenida que incluiría la realización de las siguientes pruebas:

- Contraste de error de especificación: Prueba RESET de Ramsey.

- Contraste de normalidad de los errores: Prueba de Jarque-Bera.

- Contraste de homocedasticidad: Prueba de White con términos cruzados.

- Contraste de no autocorrelación serial de los errores: Estadístico $d$ de Durbin-Watson, de ser indeterminado, se añadiría la Prueba Breusch-Godfrey LM, (Pulido San Román \& Pérez García, 2001).

- Análisis de multicolinealidad en el modelo: Factor de varianza inflada.

Una vez validados los supuestos se verificaría el valor de los coeficientes $\widehat{\beta_{p}}$ estandarizados de cada variable con el fin de evaluar los impactos que tienen cada una de ellas respecto al crecimiento económico del Ecuador.

El nivel de significancia que se utilizaría para todas las pruebas sería del 5\% y el procesamiento estadístico se realizaría utilizando el paquete econométrico Eviews.

\footnotetext{
${ }^{3}$ Mínimos cuadrados ordinarios 


\section{Resultados}

Luego de descargar las series de datos, tabularlas y graficarlas, ver la figura 1, se realizó un análisis descriptivo general de las mismas, durante este se apreció un comportamiento de largo plazo homogéneo en todas las series, todas presentan tendencia creciente con poca variabilidad lo cual es indicativo de una muy probable no estacionariedad en tendencia, asimismo, puede observarse cierta sincronización en su comportamiento de largo plazo lo que puede ser evidencia de que existe cointegración entre ellas, las pruebas formales de estacionariedad y cointegración se realizarían a continuación.

\section{Análisis de estacionariedad y cointegración}

Antes de estimar la ecuación de regresión entre las variables estudiadas se verificó la estacionariedad de las series y su orden de integración. Primeramente, se aplicó la prueba $A D F$ a cada serie en niveles, probándose en cada caso la existencia de raíz unitaria y, por tanto, la no estacionariedad de las mismas en niveles, luego se volvió a realizar el test $A D F$ a cada serie, pero en primeras diferencias, hallándose, para todas las variables, que eran estacionarias en primeras diferencias y, por tanto, integradas de 1er. orden. Dados los resultados anteriores, en ningún caso fue necesario contrastar la prueba $A D F$ con otra prueba de raíz unitaria o estacionariedad, por ejemplo, la prueba KPSS, como se había planteado en la metodología.

A partir de los resultados anteriores se hizo necesario evaluar si existía cointegración entre las variables previo a realizar la regresión con el fin de garantizar que esta no sería espuria. A este fin se realizó la prueba de Cointegración de Engle - Granger cuyo resultado, que puede ser verificado en la tabla 1, demuestra que existe cointegración entre las variables, lo que es evidencia de equilibrio a largo plazo entre las mismas y, por tanto, de que la ecuación de regresión lineal estimada entre ellas teniendo como variable dependiente al PIB no sería espuria.

\section{Tabla 1.}

Resultado de la prueba de Cointegración de Engle - Granger.

Series: PIB Inversión Importación Gasto Exportación Consumo

Sample: 19652018

Included observations: 54

Null hypothesis: Series are not cointegrated

Cointegrating equation deterministics: $\mathrm{C}$

Automatic lags specification based on Schwarz criterion (maxlag=10)

\begin{tabular}{ccccc}
\hline Dependent & tau-statistic & Prob. $^{*}$ & Z-statistic & Prob. $^{*}$ \\
\hline PIB & -5.494113 & $\mathbf{0 . 0 1 8 3}$ & -38.86386 & $\mathbf{0 . 0 1 5 9}$ \\
Inversión & -3.887266 & 0.3396 & -23.97395 & 0.3219 \\
Importación & -5.652550 & 0.0126 & -40.43162 & 0.0104 \\
Gasto & -5.090808 & 0.0443 & -34.96738 & 0.0416 \\
Exportación & -5.820645 & 0.0085 & -41.78695 & 0.0071 \\
Consumo & -5.473215 & 0.0192 & -38.63029 & 0.0169 \\
\hline
\end{tabular}

*MacKinnon (1996) p-values.

Fuente: Elaboración por los autores. 


\section{Estimación de la ecuación de regresión lineal}

Luego, a partir de los datos sin transformar correspondientes a los años 1965 hasta 2018, 54 observaciones, se obtuvo el modelo de regresión lineal múltiple por MCO en su forma funcional lineal-lineal que se muestra en la tabla 2.

Tabla 2.

Ecuación de regresión lineal obtenida con las observaciones de los años 1965 hasta 2018.

\begin{tabular}{|c|c|c|c|c|}
\hline \multicolumn{5}{|l|}{$\begin{array}{l}\text { Dependent Variable: PIB } \\
\text { Method: Least Squares } \\
\text { Sample: } 19652018 \\
\text { Included observations: } 54\end{array}$} \\
\hline $\begin{array}{c}\text { Variable } \\
\end{array}$ & Coefficient & Std. Error & t-Statistic & Prob. \\
\hline Inversión & 0.531595 & 0.074029 & 7.180895 & 0.0000 \\
\hline Importación & -0.664031 & 0.068539 & -9.688427 & 0.0000 \\
\hline Gasto & 1.546702 & 0.109373 & 14.14155 & 0.0000 \\
\hline Exportación & 0.883151 & 0.066066 & 13.36761 & 0.0000 \\
\hline Consumo & 0.989782 & 0.017309 & 57.18354 & 0.0000 \\
\hline $\mathrm{C}$ & 129412.7 & 97463.32 & 1.327809 & 0.1905 \\
\hline R-squared & 0.999895 & Mean dependent var & & 31852342 \\
\hline Adjusted R-squared & 0.999884 & S.D. dependent var & & 31566657 \\
\hline S.E. of regression & 339615.2 & Akaike info criterion & & 28.41345 \\
\hline Sum squared resid & $5.54 \mathrm{E}+12$ & Schwarz criterion & & 28.63445 \\
\hline Log likelihood & -761.1632 & Hannan-Quinn criter. & & 28.49868 \\
\hline F-statistic & 91567.89 & Durbin-Watson stat & & 1.461587 \\
\hline Prob(F-statistic) & 0.000000 & & & \\
\hline
\end{tabular}

Fuente: Elaboración por los autores.

Entonces, la ecuación de regresión estimada, a partir de la tabla anterior, fue:

$$
\widehat{P I B}=129412.70+0.99 * C+0.53 * I+1.55 * G+0.88 * X-0.66 * M
$$

Al observar la tabla 2 se aprecia que, el p_valor ${ }^{4}$ de la prueba $F$ de significancia global es significativo al igual que todos los p_valores correspondientes a las pruebas $t$ de significancia individual de cada coeficiente $\widehat{\beta_{p}}$ de la ecuación de regresión, por lo que todos ellos son significativos, probándose, por tanto, que todas las variables explicativas incluidas en el modelo estimado son determinantes para explicar la variabilidad de la variable dependiente.

\section{Validación de supuestos}

Una vez probada la significancia de las variables para el modelo obtenido se continuó validando su corrección estadística mediante la prueba de los supuestos de la regresión lineal:

1. Con el fin de determinar si la especificación lineal para las variables predictoras es la correcta se realizó la prueba RESET de Ramsey, constatándose que para combinaciones no lineales de 2 do. y 3 er. orden no existe poder explicativo por parte

\footnotetext{
${ }^{4}$ Valor de probabilidad 
de los predictores, por lo que en ambos casos hay evidencia suficiente para concluir que la especificación lineal del modelo es la adecuada.

2. Respecto a la verificación de normalidad de los errores se ejecutó la prueba de JarqueBera obteniéndose un p_valor de 0.80 que permite no rechazar la hipótesis nula de la prueba que afirma que los errores siguen una distribución normal.

3. Para comprobar si el error se mantiene constante a lo largo de las observaciones de los regresores se procedió a correr la prueba de White con términos cruzados la que dio un p_valor para el estadístico F de 0.26 , por lo que concluimos que no hay evidencia para afirmar que existe heterocedasticidad.

4. En cuanto al supuesto de no autocorrelación serial de los errores el estadístico $d$ de Durbin-Watson toma un valor de 1.46 que cae en zona de indecisión, dado que los límites $d_{L}$ y $d_{U}$ para 54 observaciones y 5 variables predictoras son 1.37 y 1.77 respectivamente, es por ello que se recurre a la prueba de Breusch-Godfrey LM comprobándose que no hay autocorrelación serial de orden 1 ni de orden 2.

5. Al efecto de probar la no existencia de multicolinealidad en el modelo, se comprueba que no existe multicolinealidad perfecta pues MCO estimó los coeficientes de regresión de las variables predictoras, ver tabla 2. En cuanto a la multicolinealidad imperfecta, se procedió al cálculo del factor de varianza inflada para cada variable independiente comprobándose que existe una alta multicolinealidad imperfecta.

Como se observa los supuestos de la regresión lineal múltiple para la ecuación obtenida quedaron validados excepto para la multicolinealidad imperfecta que es alta para el modelo obtenido, no obstante, según (Montero Granados, 2016), la multicolinealidad no sólo es normal, sino que es esperable y deseable. Es imposible que unas variables que explican y son explicadas por un fenómeno sean tan completamente independientes que no estén correlacionadas en algún grado, asimismo, (Gujarati \& Porter, 2010) expresan que, otra razón para la multicolinealidad, sobre todo en los datos de series de tiempo, puede ser que las regresoras del modelo compartan una tendencia común; es decir, que todas aumenten o disminuyan a lo largo del tiempo, que, obviamente, es el caso que se da en el conjunto de datos objeto de la investigación.

Una de las formas de reducir o eliminar la multicolinealidad sería suprimiendo alguno de los regresores, los más correlacionados entre sí, pero no consideramos pertinente esta medida, pues incurriríamos en sesgo de especificación a tenor de (Gujarati \& Porter, 2010), que plantean que si la teoría económica afirma que unas variables determinadas deben incluirse en el modelo que explica el fenómeno que se estudia, al eliminar una de esas variables se cometería el error de especificación, en este caso el método de gasto para el cálculo del PIB incluye, según la teoría, todas las variables que aparecen en el modelo y, por esa razón, no deben ser excluidas del mismo.

Además, expresa (Fernández Montt, 2006), que en presencia de alta multicolinealidad los estimadores MCO de los coeficientes de regresión siguen siendo MELI ${ }^{5}$, también (Achen, 1982) y (Wooldridge, 2010) postulan que, la multicolinealidad no viola los supuestos básicos de la regresión y que se presentarán estimaciones consistentes e insesgadas y sus errores estándar se estimarán en la forma correcta, asimismo, según (Gujarati \& Porter, 2010), con alta multicolinealidad es probable que el p_valor $t$ de uno o más coeficientes tienda a ser

\footnotetext{
${ }^{5}$ Mejores estimadores lineales e insesgados 
estadísticamente no significativo, lo cual no ocurre con el estimador hallado donde sí son muy significativos.

\section{Análisis del impacto en el PIB de cada componente de la ecuación estimada}

Finalmente, y para dar cumplimiento del objetivo general planteado en la investigación una vez que se ha estimado una ecuación estadísticamente válida y donde son significativas todas las variables que incluye el método de gasto del PIB se procede a verificar el impacto que cada variable explicativa tiene sobre el PIB, es decir, cuán importantes o no son el consumo, la inversión, el gasto del gobierno y las exportaciones netas en la determinación del crecimiento económico del Ecuador.

Para ello se calcularon los coeficientes $\widehat{\beta_{p}}$ estandarizados, que pueden ser visualizados en la tabla 3. Revisando el peso relativo a cada variable predictora podemos observar en la tabla que

los coeficientes $\widehat{\beta_{p}}$ estandarizados demuestran que en el caso que se estudia es el consumo "C" el indicador que mayor importancia o peso tiene a los efectos de explicar el PIB seguido por las exportaciones " $\mathrm{X}$ ", el gasto "G", las importaciones "M" y la inversión "I" como la de menor peso o impacto.

Tabla 3.

Coeficientes $\widehat{\beta_{p}}$ estandarizados.

Scaled Coefficients

Sample: 19652018

Included observations: 54

\begin{tabular}{cccc}
\hline Variable & Coefficient & $\begin{array}{c}\text { Standardized } \\
\text { Coefficient }\end{array}$ & $\begin{array}{c}\text { Elasticity } \\
\text { at Means }\end{array}$ \\
\hline Inversión & 0.531595 & $\mathbf{0 . 1 4 2 2 9 9}$ & 0.120677 \\
Importación & -0.664031 & $\mathbf{- 0 . 1 8 7 8 7 6}$ & -0.169171 \\
Gasto & 1.546702 & $\mathbf{0 . 2 1 7 9 2 3}$ & 0.205825 \\
Exportación & 0.883151 & $\mathbf{0 . 2 3 7 8 1 4}$ & 0.212974 \\
Consumo & 0.989782 & $\mathbf{0 . 5 9 5 6 1 0}$ & 0.625632 \\
$\mathrm{C}$ & 129412.7 & NA & 0.004063 \\
\hline
\end{tabular}

Fuente: Elaboración por los autores.

\section{Conclusiones}

Como conclusiones de la investigación se relacionan las siguientes:

El análisis de regresión sobre los datos de las variables incluidas en la ecuación de cálculo del PIB del Ecuador durante el período 1965 - 2018 demuestra que estas son significativas y presentan el tipo de relación con la variable dependiente que plantea el método. Asimismo, se demuestra que la regresión no es espuria y que todos los supuestos del modelo se cumplen, por tanto, la ecuación obtenida es estadísticamente válida y adecuada para extraer conclusiones a partir de ella.

Al analizar los coeficientes estandarizados de la ecuación de regresión estimada se confirma un supuesto teórico, según (Abel \& Bernanke, 2004), pues el componente más 
significativo de la demanda agregada, cuyo proxi en esta investigación es el PIB, es el consumo "C", vea la tabla 3.

Respecto a la interpretación concreta del resultado que muestran los coeficientes $\widehat{\beta_{p}}$ estandarizados que nos permiten cuantificar el impacto que cada variable del modelo tiene en el crecimiento económico del país se pueden inferir varias conclusiones relevantes para los responsables de la política económica del Ecuador:

1. El consumo es fundamental para generar demanda agregada y, por consiguiente, crecimiento económico.

2. Respecto a las exportaciones y el gasto del gobierno puede verse el peso significativo, y casi del mismo nivel, que ambos indicadores tienen respecto al crecimiento económico.

3. El impacto de la importación, aunque en cuanto a peso absoluto queda en cuarto lugar, no deja de ser importante por cuanto su incidencia respecto al crecimiento del PIB es negativa.

4. En el caso de la inversión esta no se comporta como el motor fundamental del crecimiento económico que debería ser, lo que demuestran los datos empíricos, siendo el indicador de menor impacto en el PIB nacional, esta situación es muy negativa para la economía.

Respecto a las recomendaciones derivadas de los hallazgos de la investigación, se enumeran las siguientes:

1. Dada la importancia fundamental del consumo de las familias para el crecimiento, las políticas fiscales, monetarias o de empleo que tiendan a reducirlo van a tener un impacto notablemente negativo en el crecimiento económico de la nación Es importante, que desde el gobierno se planteen políticas e iniciativas, que articulen el sector público y el privado, de manera tal que se fomente el empleo adecuado y la estabilidad laboral, la economía en general saldrá fortalecida a partir del crecimiento del consumo que sería impulsado por el crecimiento del empleo adecuado. La teoría macroeconómica es clara al respecto, la renta disponible de las familias se dedica al consumo y al ahorro, si se logra fomentar un ingreso estable para las personas va a crecer el consumo necesariamente.

2. Teniendo en cuenta el rol de las exportaciones para el crecimiento económico ecuatoriano los decisores de política económica deberán prestar atención al desarrollo de acciones que fortalezcan e incentiven al sector exportador y propendan a generar una balanza comercial superavitaria.

3. Por otra parte, con el debido control del déficit presupuestario, las políticas que generen gasto del gobierno, especialmente aquel que se destine a infraestructura e inversión es conveniente para impulsar el crecimiento.

4. Respecto al impacto negativo de las importaciones sobre el crecimiento, deben adoptarse políticas orientadas a reducir las importaciones sustituyendo materias primas e insumos importados por producción nacional lo cual va a resultar favorable para el crecimiento de la economía.

5. En referencia al pobre desempeño y aporte de la inversión al crecimiento, esta es una situación que debe ser revertida y que muestra que esta es una variable donde hay importantes reservas que explotar a partir de desarrollar políticas que la incentiven de manera vigorosa y sostenida, en esto la empresa privada juega el papel determinante, por tanto, desde el gobierno, y mediante una legislación que garantice, ofrezca estabilidad y formalice un 
marco regulatorio adecuado para la misma es necesario que los empresarios privados ecuatorianos se comprometan con su país y pongan como principal destino de sus recursos al Ecuador. También debería fomentarse, con las políticas adecuadas, la inversión extranjera directa, la cual es aún baja en el Ecuador comparada con otros países de la región latinoamericana.

\section{Bibliografía}

Abel, A. B., \& Bernanke, B. S. (2004). Macroeconomía, 4ta. Ed. Madrid: Pearson Educación. Achen, C. H. (1982). Interpreting and Using Regression. Beverly Hills: Sage.

Anderson, D., Sweeney, D., \& Williams, T. (2008). Estadística para administración y economía. 10ma. Ed. México D.F.: Cengage Learning Editores, S.A.

Banco Central del Ecuador. (2020). Estadísticas del sector real. Obtenido de Información Económica: https://contenido.bce.fin.ec/documentos/Administracion/CuentasNacionalesAnuales.html

Case, K. E., \& Fair, R. C. (2008). Principios de macroeconomía, 8va. Ed. México D. F.: Pearson Educación.

Court, E., \& Williams, E. (2011). Estadísticas y econometría financiera, 1ra. Ed. Buenos Aires: Cengage Learning Argentina.

Dornbusch, R., Fischer, S., \& Startz, R. (2009). Macroeconomía, 10ma. Ed. México D. F.: McGraw-Hill/Interamericana Editores, S.A. de C.V.

Fernández Montt, R. A. (2006). Regresión lineal. Multicolinealidad perfecta. Obtenido de Enciclopedia multimedia virtual interactiva: http://www.eumed.net/cursecon/medir/rfmmultico.htm

Gastón Lorente, L. (2019). Cómo calcular el PIB: Tres métodos. Obtenido de BBVA: https://www.bbva.com/es/bbva-patrocina-el-almuerzo-inaugural-de-la-cumbre-del-climadisenado-por-los-hermanos-roca/

González González, M., Pérez Zabaleta, A., Castejón Montijano, R., Méndez Pérez, E., Martínez Merino, J., Gómez Barroso, J., \& Mochón Sáez, A. (2009). Introducción a la economía, 2da. Ed. Madrid: Pearson Educación.

Gujarati, D. N., \& Porter, D. C. (2010). Econometría. 5ta. Ed. México, D. F.: McGraw Hill Educación.

Jahan, S., Saber Mahmud, A., \& Papageorgiou, C. (09 de 2014). ¿Qué es la economía keynesiana? Obtenido de Revista Finanzas y Desarrollo. Fondo Monetario Internacional.: https://www.imf.org/external/pubs/ft/fandd/spa/2014/09/pdf/basics.pdf

Kiziryan, M. (2019). Demanda agregada. Obtenido de Economipedia: https://economipedia.com/definiciones/demanda-agregada.html

Lind, D., Marchal, W., \& Wathen, S. (2012). Estadística aplicada a los negocios y la economía. 15ta. Ed. México D.F.: McGraw Hill/Interamericana Editores S.A. de C.V.

Mahadeva, L., \& Robinson, P. (2009). Prueba de raíz unitaria para ayudar a la construcción de un modelo. Obtenido de Centro de Estudios Monetarios Latinoamericanos: https://www.cemla.org/PDF/ensayos/pub-en-76.pdf

Mankiw, N. G. (2014). Macroeconomía, 8va. Ed. Barcelona: Antoni Bosch editor, S.A.

Montero Granados, R. (2016). Modelos de regresión lineal múltiple. Documentos de Trabajo en Economía Aplicada. Universidad de Granada. España. 
Parkin, M., Esquivel, G., \& Muñoz, M. (2007). Macroeconomía. Versión para Latinoamérica, 7ma. Ed. México: Pearson Educación.

Pulido San Román, A., \& Pérez García, J. (2001). Modelos econométricos. Madrid: Ediciones Pirámide.

Samuelson, P. A., \& Nordhaus, W. D. (2010). Macroeconomía con aplicaciones a Latinoamérica, 19na. Ed. México D. F.: McGraw Hill Educación.

Wooldridge, J. M. (2010). Introducción a la econometría. Un enfoque moderno. 4ta. Ed. México, D. F.: Cengage Learning. 